\title{
Utilização de lignosulfonato e adesivo Fenol-Formaldeído na produção de painéis aglomerados
}

\author{
Using lignosulfonate and Phenol-Formaldeyde \\ adhesive in particleboard manufacturing
}

\author{
Marcelly Alves da Silva ${ }^{1}$, Pablo Vieira Dos Santos ${ }^{1}$, Gilmar Correia Silva ${ }^{2}$, \\ Roberto Carlos Costa Lelis ${ }^{3}$, Alexandre Miguel Do Nascimento ${ }^{4}$ e Edvá Oliveira Brito ${ }^{3}$
}

\begin{abstract}
Resumo
O objetivo deste estudo foi avaliar a viabilidade da utilização de lignosulfonato de cálcio e magnésio (LS) na produção de painéis aglomerados de Pinus caribaea var. caribaea, na forma pura e em misturas com fenol-formaldeído (FF). De modo a avaliar o efeito da substituição de FF por LS sobre as propriedades físicas e mecânicas, foram produzidos painéis com diferentes concentrações desses adesivos. $O$ delineamento experimental foi constituído de duas fases. A primeira fase foi realizada sob a temperatura de $160^{\circ} \mathrm{C} \mathrm{e} \mathrm{tem-}$ po de prensagem de 10 minutos, com as seguintes composições: $100 \%$ de FF, e as substituições por LS nas proporções de $20,40,60,80$ e $100 \%$, com três repetições cada. Na segunda fase, os três tratamentos da primeira fase com desempenho inferior nas propriedades mecânicas foram testados na temperatura de $180^{\circ} \mathrm{C}$ em tempos de prensagem de 5 e 7 minutos. Os resultados médios da densidade básica e aparente da madeira foram de 0,54 e $0,60 \mathrm{~g} / \mathrm{cm}^{3}$, respectivamente. Na segunda fase, o aumento da temperatura e a diminuição do tempo de prensagem não foram suficientes para consolidar o painel, não sendo possível analisar os resultados desta fase. Com relação ao inchamento em espessura e absorção de água, à medida que aumentou a proporção de lignosulfonato na mistura elevaram-se também esses valores. Em até $80 \%$, a substituição do adesivo fenol-formaldeído por lignosulfonato foi satisfatória no atendimento a NBR 14810-2 (ABNT,2013) para propriedades mecânicas. O lignosulfonato apresentou eficiência na utilização como adesivo na produção de painéis aglomerados em misturas com fenol-formaldeído, evidenciando que esta é uma alternativa à utilização de adesivos sintéticos.
\end{abstract}

Palavras-chave: lignosulfonato; fenol-formaldeído; Pinus caribaea.

\begin{abstract}
The purpose of this study was to evaluate the feasibility of using calcium and magnesium lignosulfonate (LS) in the production of Pinus caribaea var. caribaea particleboard panels, used pure and in mixtures with phenol-formaldehyde (FF). In order to evaluate the effect of FF replaced by LS on the physical and mechanical properties, panels were produced with different concentrations of these adhesives. The experiment consisted of two phases: The first phase was performed under temperature of $160^{\circ} \mathrm{C}$ and pressing time of 10 minutes with the following compositions: $100 \% \mathrm{FF}$, and LS substitutions in the proportions of $20,40,60,80$ and $100 \%$, with three replications each. In the second phase, the first phases of the three treatments with lower performance in the mechanical properties were tested at a temperature of $180^{\circ} \mathrm{C}$ and up to 7 minutes. The results of basic and apparent wood density were 0.54 and $0.60 \mathrm{~g} / \mathrm{cm}^{3}$, respectively. In the second stage, the temperature increase and decrease of the pressing time were not sufficient to consolidate the panel, rendering the analysis of the results of this phase not possible. As for thickness swelling and water absorption, increases in the proportion of lignosulfonate in the mixture also raised up those values. The replacement of the phenol-formaldehyde adhesive by lignosulfonate was satisfactory, attending the specific norm for mechanical properties by $80 \%$. Lignosulfonate showed efficiency as use as an adhesive in the production of particleboard in mixtures with phenol formaldehyde, suggesting that this is an alternative to the use of synthetic adhesives.
\end{abstract}

Keywords: lignosulfonate; phenol formaldehyde; Pinus caribaea.

\footnotetext{
${ }^{1}$ Mestrando em Ciências Ambientais e Florestais. UFRRJ - Universidade Federal Rural do Rio de Janeiro / Instituto de Florestas. Rodovia BR-465, Km 7 - 23890-000 - Seropédica, RJ, Brasil. E-mail: marcellyufrri@hotmail.com; pabloufrri@hotmail.com.

2Professor Associado do Departamento de Fitotecnia e Zootecnia. UESB - Universidade Estadual do Sudoeste da Bahia. Estrada Bem Querer - 45031300 - Vitória da Conquista, BA. Brasil. E-mail: gcsflorestal@gmail.com; edva@ufrri.br.

3Professor Titular do Departamento de Produtos Florestais. UFRRJ - Universidade Federal Rural do Rio de Janeiro / Instituto de Florestas. Rodovia BR-465, Km 7 - 23890-000 - Seropédica, RJ, Brasil. E-mail: lelis@ufrrj.br; amn@ufrrj.br.

${ }_{4}^{4}$ Professor Adjunto do Departamento de Produtos Florestais. UFRRJ - Universidade Federal Rural do Rio de Janeiro / Instituto de Florestas. Rodovia BR-465, Km 7 - 23890-000 - Seropédica, RJ, Brasil. E-mail: adva@ufrrj.br;
}

Sci. For., Piracicaba, v. 45, n. 115, p. 423-433, set. 2017 DOI: dx.doi.org/10.18671/scifor.v45n115.01 


\section{INTRODUÇÃO}

A indústria de painéis aglomerados surgiu no início da década de 1940, na Alemanha, com a finalidade de aproveitar os resíduos industriais e das serrarias, uma vez que havia dificuldade para a produção de lâminas de boa qualidade para a fabricação de compensados (DONATI, 2010). No Brasil, em 1966, os painéis de madeira aglomerada começaram a ser produzidos pela indústria iniciada pelo grupo francês Louis Dreiffus, que criou a empresa Placas do Paraná, em Curitiba, devido à abundância de resíduos produzidos na região Sul pela indústria moveleira local (MATTOS et al. 2008).

Os painéis de madeira constituem uma alternativa à madeira maciça, além de diminuir o custo do produto, os painéis de madeira possuem as vantagens de amenizar a anisotropia e a instabilidade dimensional, e ainda melhorar as propriedades isolantes, térmicas e acústicas. Adicionalmente, suprem a necessidade do uso da madeira serrada e ampliam a sua superfície útil, através da expansão de uma de suas dimensões (a largura), para, assim, otimizar a sua aplicação (REMADE, 2004). Melo et al. (2010) citam que os painéis aglomerados podem ser produzidos a partir de qualquer material ligno-celulósico, desde que confira resistência física, mecânica e biológica adequadas aos painéis aglomerados.

Nas indústrias de painéis aglomerados, as resinas tradicionalmente utilizadas são as fenólicas e uréicas, sendo estas últimas de baixa resistência à umidade (ROFFAEL, 1982), ambas obtidas pela condensação de formaldeído com fenol ou ureia, respectivamente. A crise do petróleo de 1970 intensificou a preocupação em substituir estes derivados petroquímicos na indústria de painéis de madeira (CHOW, 1983; PIZZI; MITTAL, 1994), adicionalmente, a possibilidade de escassez desses recursos impulsiona cada vez mais o desenvolvimento de novos produtos, oriundos de fontes renováveis (ALEMDAR; SAIN, 2008).

Neste sentido, um polímero natural que vem ganhando destaque é o lignosulfonato, também chamado de lignina sulfonada. Trata-se de um subproduto rico em lignina gerado a partir do processo de polpação da madeira pelos processos ácidos sulfito ou bissulfito. De acordo com o processo de polpação, podem ser obtidos lignosulfonatos de cálcio, sódio, magnésio e amônio (RODRÍGUEZ, 2005). A este respeito, a substituição parcial de fenol por lignina tem sido apresentada como a alternativa mais atraente econômica e ambientalmente, devido à semelhança estrutural entre este polímero natural de caráter fenólico com resinas de fenol-formaldeído (FORSS; FUHRMANN, 1979).

Considerando-se o exposto, o objetivo deste trabalho foi avaliar a viabilidade de utilização de lignosulfonatos como adesivo e o efeito da substituição do adesivo fenol-formaldeído por diferentes proporções de lignosulfonato sobre as propriedades físicas e mecânicas dos painéis aglomerados.

\section{MATERIAL E MÉTODOS}

\section{Obtenção da madeira para a confecção dos painéis}

Neste estudo foram utilizadas duas árvores da espécie Pinus caribaea var. caribaea provenientes de um plantio localizado na Universidade Federal Rural do Rio de Janeiro (UFRRJ), no município de Seropédica - RJ. O povoamento florestal possuía idade estimada de 27 anos no momento do abate. Fez-se uma seleção prévia visando identificar as árvores aptas para o abate, ou seja, as que apresentavam, visualmente, um bom aspecto fitossanitário. Posteriormente, fez-se o abate das árvores aleatoriamente. As árvores apresentaram 20,5 cm e 21,2 cm de DAP.

\section{Determinação da densidade básica e aparente da madeira}

A partir do material coletado, foram utilizadas amostras radiais de $2,5 \mathrm{~cm}$ de espessura ao longo do tronco nas posições 0\% (base), 25\%, 50\%, 75\% e 100\% (topo) e à altura do DAP (1,30m do solo) para determinação da densidade básica. A densidade básica da madeira foi realizada pelo método de imersão em água, conforme descrito por Vital (1984). Nesse método, a amostra saturada, foi submersa em água, e a variação de massa foi registrada em uma balança de precisão, fornecendo o volume da amostra (Princípio de Arquimedes). Posteriormente, essa amostra foi seca em estufa 
até a estabilização da massa. Determinou-se em seguida, a sua massa seca, estabelecendo-se assim a relação massa seca/volume saturado.

Adicionalmente, foram retiradas amostras para determinação da densidade aparente a $12 \%$ de teor de umidade nas dimensões 2 × 2 × $3 \mathrm{~cm}$, nos sentidos radial, tangencial e longitudinal, respectivamente. Essas amostras foram acondicionadas em sala de climatização e após a estabilização determinou-se o volume, utilizando o método de deslocamento em mercúrio (Hg), citado por Vital (1984). Para tanto, as amostras foram pesadas em balança de precisão e com auxílio de uma haste de ferro, imersas, uma a uma, em um becker com mercúrio. Em seguida, registrou-se a massa do líquido deslocado e com base na densidade do Hg calculou-se o volume da amostra. Assim, com base na massa e volume de cada amostra, calculou-se a densidade aparente.

\section{Obtenção dos adesivos}

O adesivo fenol-formaldeído (FF) e o lignosulfonato (LS) utilizados neste estudo foram gentilmente cedidos pelas empresas Momentive e Melbar, respectivamente.

O lignosulfonato, respeitando as considerações do fabricante, necessitou ser diluído na proporção de $45 \%$, sendo $45 \%$ de lignosulfonato e o restante em água deioneizada, pois o mesmo é comercializado em pó, ao contrário do adesivo fenol-formaldeído, em estado líquido.

Devido ao contraste entre o $\mathrm{pH}$ do fenol-formaldeído, bastante alcalino $(\mathrm{pH}=11,25)$, e o $\mathrm{pH}$ ácido do lignosulfonato ( $\mathrm{pH}=2,82)$, se fez necessária a substituição de parte deste último por hidróxido de sódio, de modo que a mistura entre os dois componentes se tornasse menos viscosa, permitindo a aspersão dos mesmos pela pistola. Esta substituição foi realizada para os tratamentos que continham a mistura de ambos componentes.

\section{Produção de painéis aglomerados}

Os painéis foram confeccionados visando atender a NBR 14810-2 (2013), sendo classificados como painéis de partículas de média densidade Tipo P3 - painéis não estruturais para uso em condições úmidas.

Logo após o abate, as árvores foram serradas em toretes e por sua vez foram segmentados em discos com aproximadamente 5,0 cm de espessura. Os discos foram seccionados em três partes para serem levados ao gerador de partículas, originando partículas tipo "strand". Estas possuem comprimento variando de 12,7 a 76,2 $\mathrm{mm}$, largura 6,4 a 25,4 $\mathrm{mm}$ e espessura de 0,25 a 0,64 $\mathrm{mm}$. Em seguida foram levadas ao moinho de martelo, gerando partículas do tipo "flake", com comprimento entre 25 e $100 \mathrm{~mm}$, largura entre 10 e $25 \mathrm{~mm}$, e espessura variando entre 0,2 e 0,4 mm.

As partículas foram secas ao ar e posteriormente homogeneizadas por meio de um peneirador mecânico composto por duas peneiras, a primeira de $4,1 \mathrm{~mm}$ reteve as partículas mais grossas, e a segunda de 0,49 $\mathrm{mm}$ reteve as partículas desejáveis e já os finos foram sendo depositados no fundo do peneirador.

Após serem peneiradas, as partículas foram transportadas para uma estufa com circulação de ar a fim de atingirem umidade média em torno de $5 \%$.

As partículas foram postas em um tambor rotatório para que enfim, o adesivo fosse justaposto às mesmas por meio de uma pistola pneumática, de modo mais uniforme possível. Logo depois, fez-se o colchão de partículas utilizando-se uma forma de madeira com dimensões 40 x 40 x $20 \mathrm{~cm}$, visando a obtenção de painéis de 40 x 40 x 1,27 cm. Em seguida, o colchão foi levado à prensa hidráulica com pressão da prensagem de 3,92 MPa, visando atender uma densidade nominal de 0,70 $\mathrm{g} / \mathrm{cm}^{3} \mathrm{em}$ cada painel. Os painéis foram esquadrejados nas dimensões 30 x $30 \mathrm{~cm}$ e acondicionados em sala climatizada com umidade relativa em torno de $65 \pm 5 \%$ e temperatura de $20 \pm 3{ }^{\circ} \mathrm{C}$, até atingir a umidade de equilíbrio.

\section{Delineamento experimental}

A produção de painéis utilizando adesivo à base de lignosulfonato e fenol-formaldeído se deu em duas fases. Para os tratamentos foram feitas três repetições cada. Os tratamentos são representados nas Tabelas 1 e 2. 
1)Primeira fase:

Tabela 1. Composição dos tratamentos para temperatura $160^{\circ} \mathrm{C}$.

Table 1. Composition treatments at $160^{\circ} \mathrm{C}$ temperature.

\begin{tabular}{|c|c|c|c|c|}
\hline \multirow{2}{*}{ Tratamento } & \multicolumn{2}{|c|}{ Composição do Adesivo (\%) } & \multirow{2}{*}{$\begin{array}{c}\text { Tempo de Prensagem } \\
\text { (min) }\end{array}$} & \multirow[b]{2}{*}{$\mathrm{T}\left({ }^{\circ} \mathrm{C}\right)$} \\
\hline & Fenol Formaldeído (FF) & Lignosulfonato (LS) & & \\
\hline T1 & 100 & 0 & \multirow{6}{*}{10} & \multirow{6}{*}{160} \\
\hline $\mathrm{T} 2$ & 80 & 20 & & \\
\hline T3 & 60 & 40 & & \\
\hline T4 & 40 & 60 & & \\
\hline T5 & 20 & 80 & & \\
\hline T6 & 0 & 100 & & \\
\hline
\end{tabular}

Em que: $\mathrm{T}=$ Temperatura de prensagem em ${ }^{\circ} \mathrm{C}$.

Nesta fase o delineamento experimental foi realizado inteiramente ao acaso. Os painéis foram confeccionados compondo seis tratamentos com três repetições cada, totalizando 18 painéis.

De acordo com o desempenho dos painéis, elaborou-se a segunda fase do delineamento experimental, selecionando-se os que obtiveram resultados menos satisfatórios junto ao atendimento dos ensaios tecnológicos e de inchamento da NBR 14810 (ABNT, 2013).

2)Segunda fase:

Tabela 2. Composição dos tratamentos para variações de adição lignosulfonato e fenol formaldeído, com tempo de prensagem de 5 e 7 minutos e temperatura de $180^{\circ} \mathrm{C}$.

Table 2. Composition treatments with addition variations of lignosulphonate and phenol formaldehyde at $180 \circ \mathrm{C}$ temperature, 5 and 7 minute pressing time.

\begin{tabular}{lcccc}
\hline \multirow{2}{*}{ Tratamento } & \multicolumn{2}{c}{ Composição do Adesivo (\%) } & $\begin{array}{c}\text { Tempo de Prensagem } \\
\text { (min) }\end{array}$ & T ( $\left.{ }^{\circ} \mathrm{C}\right)$ \\
\cline { 2 - 3 } & Fenol Formaldeído (FF) & Lignosulfonato (LS) & 5 & \\
\hline T4 & 40 & 60 & 5 \\
T5 & 20 & 80 & & \\
T6 & 0 & 100 & 7 \\
T4 & 40 & 60 & \\
T5 & 20 & 80 & \\
T6 & 0 & 100 & \\
\hline
\end{tabular}

Em que: $\mathrm{T}=$ Temperatura de prensagem em ${ }^{\circ} \mathrm{C}$.

Nesta segunda fase, procurou-se otimizar o tempo da produção dos painéis, verificando se para os tratamentos mencionados seria possível melhoras nas propriedades físicas e mecânicas com a diminuição do tempo de prensagem e aumento da temperatura.

O delineamento constituído foi inteiramente casualizado com arranjo fatorial $3 \times 2$, ou seja, 3 composições de adesivos, 2 tempos de prensagem, com três repetições em cada tratamento, totalizando 18 painéis.

\section{Avaliação das propriedades físicas e mecânicas dos painéis}

Os ensaios físicos realizados foram: Umidade de equilíbrio (U) - NBR 14810 (ABNT, 2013); Inchamento em Espessura (IE) após 2 h e 24 h - NBR 14810 (ABNT, 2013); Absorção de Água (AA) após 2 h e 24 h; e Densidade do painel (D) - NBR 14810 (ABNT, 2013).

Os ensaios mecânicos foram realizados de acordo com a NBR 14810 (ABNT, 2013): Resistência à Flexão Estática (Módulo de Ruptura - MOR e Módulo de Elasticidade - MOE) e Resistência à Tração Perpendicular ao Plano dos Painéis (Ligação Interna - LI).

\section{Análise dos dados}

As densidades básica e aparente da madeira, bem como as propriedades dos adesivos, foram analisadas por meio de estatística descritiva.

Já para a análise dos ensaios físicos e mecânicos dos painéis, utilizou-se o programa Statistica 10. Atendendo as pressuposições da análise de variância, normalidade e homogeneidade de variâncias, foi utilizado o Teste de Tukey ao nível de 5\% de significância. Não atendendo as pressuposições, aplicou-se o teste não paramétrico de Kruskal-Wallis para comparações de médias. 


\section{RESULTADOS E DISCUSSÃO}

\section{Densidade Básica e Aparente da Madeira}

Os valores médios de densidade básica e densidade aparente da madeira de Pinus caribaea são apresentados na Tabela 3:

Tabela 3. Médias das densidades básica e aparente da madeira de Pinus caribaea com 27 anos.

Table 3. Averages of basic and apparent densities of Pinus caribaea at 27 years.

\begin{tabular}{ccc}
\hline Densidade Básica & Densidade Aparente \\
\hline & $\left(\mathbf{g} / \mathbf{c m}^{3}\right)$ & \\
\hline 0,54 & 0,60 \\
$(9,21)^{1}$ & $(8,56)^{1}$ \\
\hline
\end{tabular}

'Coeficiente de Variação.

Maloney (1993) cita que a indústria normalmente utiliza madeiras com densidades básicas até $0,60 \mathrm{~g} / \mathrm{cm}^{3}$ para produção de painéis. Observa-se que o valor médio encontrado para a densidade básica da madeira encontra-se dentro da faixa sugerida pelo autor.

Cabral et al. (2007) produziram painéis aglomerados a partir de misturas de partículas de Pinus elliottii e obtiveram valores de densidades básicas médias das madeiras de $0,45 \mathrm{~g} / \mathrm{cm}^{3}$.

Iwakiri et al. (2001) utilizaram cinco espécies de pinus tropicais de 10 anos, com e sem mistura entre as espécies para comparar as propriedades tecnológicas dos painéis aglomerados. Dentre estas, estava presente o Pinus caribaea, com densidade aparente média de $0,38 \mathrm{~g} / \mathrm{cm}^{3}$, que por sua vez produziram painéis com $0,65 \mathrm{~g} / \mathrm{cm}^{3}$ de densidade aparente média. Verifica-se que a densidade aparente citada pelo autor é bem inferior à encontrada neste estudo, evidenciando o que vários estudos mostram, entre 0,35 a $0,60 \mathrm{~g} / \mathrm{cm}^{3}$ é a faixa de densidade para a utilização da madeira para produção de painéis aglomerados de qualidade.

\section{Avaliação das Propriedades Físicas e Mecânicas dos Painéis}

Na primeira fase, as propriedades tecnológicas dos painéis foram analisadas de acordo com os requisitos da NBR 14810-2 (ABNT, 2013), enquadrando-os como painéis não estruturais para uso em condições úmidas (Tipo P3), em faixa de espessura nominal entre 6 a $13 \mathrm{~mm}$.

Na segunda fase do delineamento experimental, não foi possível a consolidação dos painéis em nenhuma das composições dos tratamentos para ambos os tempos de prensagem a temperatura de $180^{\circ} \mathrm{C}$, pois bolhas de ar se formaram no centro de todos os painéis e as bordas esfacelaram, não sendo possível dar prosseguimento as análises para esta fase. Este comportamento foi verificado por Silva (2015), ao fabricar painéis com mistura do adesivo ureia-formaldeído e lignosulfonato, nos mesmos tempos de prensagem desta segunda fase, a temperaturas de 160 e $180^{\circ} \mathrm{C}$. Este fato ocorreu, provavelmente, devido ao curto tempo de prensagem, impossibilitando a evaporação de toda água presente na composição do adesivo.

\section{Avaliação da densidade aparente e da umidade dos painéis}

A Tabela 4 apresenta os valores médios de densidade aparente dos painéis fabricados.

Tabela 4. Valores médios de densidade aparente e teor de umidade dos painéis fabricados para os diferentes tratamentos. Table 4. Mean values of apparent density and moisture content of the manufactured panels for different treatments.

\begin{tabular}{|c|c|c|c|c|}
\hline \multirow{2}{*}{ Tratamento } & \multicolumn{2}{|c|}{ Composição do Adesivo (\%) } & \multirow{2}{*}{ Densidade aparente $\left(\mathrm{g} / \mathrm{cm}^{3}\right)$} & \multirow{2}{*}{ Teor de Umidade (\%) } \\
\hline & FF & LS & & \\
\hline $\mathrm{T1}$ & 100 & 0 & 0,84 a $(6,99)$ & 11,11 a $(2,57)$ \\
\hline T2 & 80 & 20 & 0,83 a $(7,69)$ & 10,89 a $(3,16)$ \\
\hline T3 & 60 & 40 & 0,88 a $(6,63)$ & $10,10 \mathrm{~b}(1,38)$ \\
\hline T4 & 40 & 60 & 0,89 a $(4,21)$ & $10,03 \mathrm{~b}(2,15)$ \\
\hline T5 & 20 & 80 & 0,84 a $(6,41)$ & $10,20 \mathrm{~b}(1,42)$ \\
\hline T6 & 0 & 100 & 0,84 a $(5,19)$ & $10,31 \mathrm{~b}(1,83)$ \\
\hline
\end{tabular}

Em que: médias seguidas pela mesma letra não diferem significativamente entre si pelo teste Tukey ao nível de $5 \%$ de significância. Valores entre parênteses referem-se ao Coeficiente de Variação. 
Dentre os parâmetros de produção dos painéis, a densidade desejada para o processo de produção constava como $0,70 \mathrm{~g} / \mathrm{cm}^{3}$, mas pode-se observar que os valores obtidos foram substancialmente superiores, atingindo $0,85 \mathrm{~g} / \mathrm{cm}^{3}$ de densidade média entre todos os tratamentos. Hillig (2000) afirma que diversos fatores podem ser os responsáveis por provocar esta modificação, como a distribuição manual das partículas para formação do colchão e a possível diferença de densidade básica da matéria-prima entre partículas da mesma madeira.

A partir da determinação da densidade dos painéis e da densidade da madeira foi possível inferir sobre outro parâmetro do processo de produção, a razão de compactação. Desta forma, obteve-se razão de compactação de cerca de 1,42. O valor obtido é superior a 1,3, considerado por Moslemi (1974), Maloney (1993) e Iwakiri (2005) o mínimo para que ocorra a compactação suficiente do colchão de partículas para a formação de um painel com densidade mínima. Isso permite assegurar que houve área de contato suficiente entre as partículas durante a operação de prensagem, a fim de se obter um painel de qualidade.

Trianoski et al. (2011) produziram painéis aglomerados de Pinus taeda em mistura com cinco espécies de alto crescimento. De modo a avaliar se houve contato suficiente entre as partículas, obtiveram a razão de compactação de cada um dos tratamentos estudados, encontrando um valor médio $(1,7)$ ligeiramente superior ao encontrado neste estudo. No entanto, Moslemi (1974) e Maloney (1993) afirmam que o valor máximo aceitável para que ocorra ligação adesiva entre as partículas é 1,6.

A análise estatística demonstra que houve diferença significativa entre os teores de umidade de equilíbrio médio dos tratamentos; no entanto, os valores são bastante similares entre si, apresentando uma variação entre o valor de máximo e mínimo de 1,08 \%.

Rosa e Hillig (2014) avaliaram a viabilidade de uso do lignosulfonato de amônio como adesivo na produção de painéis aglomerados de Pinus sp., utilizado puro ou em mistura com ureia-formaldeído e tanino-formaldeído, e encontraram diferenças significativas no teor de umidade entre todos os sete tratamentos estudados, com variações de cerca de 3,03\%.

\section{Avaliação do inchamento em espessura e absorção de água}

Os dados relativos ao Inchamento em Espessura foram avaliados por meio do teste de Kruskal-Wallis são apresentados na Tabela 5.

Tabela 5. Valores médios de Inchamento em Espessura dos painéis aglomerados após 2 e 24 h de imersão em água. Table 5. Mean values of thickness swelling of particleboard after 2 and $24 \mathrm{~h}$ of immersion in water.

\begin{tabular}{lcccc}
\hline \multirow{2}{*}{ Tratamento } & \multicolumn{2}{c}{ Composição do Adesivo (\%) } & \multicolumn{2}{c}{ Inchamento em espessura (\%) } \\
\cline { 2 - 5 } & FF & LS & 2 horas & 24 horas \\
\hline T1 & 100 & 0 & $6,59 \mathrm{~b}(13,13)$ & $11,65 \mathrm{~d}(9,33)$ \\
T2 & 20 & $8,83 \mathrm{~b}(22,00)$ & $15,32 \mathrm{~cd}(19,18)$ \\
T3 & 40 & 40 & $10,77 \mathrm{bc}(29,00)$ & $20,98 \mathrm{bc}(35,61)$ \\
T4 & 60 & $18,93 \mathrm{ac}(49,33)$ & $25,59 \mathrm{ab}(44,73)$ \\
T5 & 40 & 80 & $30,87 \mathrm{a}(57,26)$ & $50,96 \mathrm{ab}(61,40)$ \\
T6 & 20 & 100 & $142,7 \mathrm{a}(73,00)$ & $168,24 \mathrm{a}(71,00)$ \\
\hline
\end{tabular}

Em que: médias seguidas pela mesma letra numa mesma coluna não diferem significativamente entre si pelo teste de Kruskal-Wallis. Valores entre parênteses referem-se a classificação das médias conforme Teste de Kruskal Wallis.

De maneira geral, observou-se que a substituição de lignosulfonato por fenol-formaldeído influenciou substancialmente no Inchamento em Espessura após 2h, de modo que, quanto maior é a proporção de lignosulfonato, maior é o Inchamento em Espessura. O mesmo comportamento foi observado nos valores de Inchamento em Espessura após $24 \mathrm{~h}$.

Não houve diferença significativa nos valores de Inchamento em Espessura após 2 horas entre os painéis fabricados com fenol-formaldeído puro (T1) e os painéis fabricados com até $40 \%$ de substituição pelo lignosulfonato (T2 e T3). Após 24h, somente não houve diferença entre fenol-formaldeído puro (T1) e a $20 \%$ de substituição (T2).

Ao comparar os valores obtidos no Inchamento em Espessura a $24 \mathrm{~h}$ com o estabelecido na NBR 14810 para painéis Tipo P3, somente os tratamentos T1 e T2 atenderam aos requisitos que foram apresentados na Tabela 3. 
Os maiores valores de Inchamento em Espessura foram encontrados nos painéis fabricados exclusivamente com adesivo à base de lignosulfonato. Não se deve deixar de mencionar que o lignosulfonato foi modificado com solução de hidróxido de sódio, que é muito higroscópico, ocasionando a obtenção de altos valores de inchamento.

Çetin e Özmen (2003) desenvolvendo uma resina fenólica a partir da substituição parcial, de 20 e 30\%, de fenol-formaldeído por lignina organosolv modificada na produção de aglomerados de Pinus sp., obtiveram enquadramento junto às normas europeias, bem como atenderiam a NBR 14810 (ABNT, 2013), pois obtiveram Inchamento em Espessura após 24 h de 12,9\% (substituição de 20\%) e 14,7\% (substituição de 30\%). Estes dados demonstram forte similaridade com os resultados obtidos neste estudo, dado o enquadramento à norma pelo T2, e aproximação do limite no T3, em que é substituído 40\% de fenol-formaldeído.

Rosa e Hillig (2014) ao avaliarem as propriedades dos painéis que continham lignosulfonato de amônio puro, obtiveram valor médio de Inchamento em Espessura para 2 h $(19,8 \%)$ substancialmente inferior ao encontrado neste estudo. No entanto, para $24 \mathrm{~h}$ o valor encontrado pelos autores foi somente cerca de $19 \%$ menor se comparado ao encontrado neste trabalho.

A Tabela 6 apresenta os valores médios de Absorção de Água após 2 e 24 h para os painéis produzidos com as diferentes composições adesivas.

Tabela 6. Valores médios de Absorção de Água dos painéis aglomerados após 2 e 24 h de imersão em água. Table 6. Mean values of water absorption of particleboard after 2 and $24 \mathrm{~h}$ of immersion in water.

\begin{tabular}{lcccc}
\hline \multirow{2}{*}{ Tratamento } & \multicolumn{2}{c}{ Composição do Adesivo (\%) } & \multicolumn{2}{c}{ Absorção de Água (\%) } \\
\cline { 2 - 5 } & FF & LS & 2 horas & 24 horas \\
\hline T1 & 100 & 0 & $30,14 \mathrm{c}(17,00)$ & $41,77 \mathrm{c}(14,73)$ \\
T2 & 80 & 20 & $35,21 \mathrm{bc}(25,45)$ & $45,89 \mathrm{c}(21,18)$ \\
T3 & 60 & 40 & $38,96 \mathrm{bc}(29,3)$ & $52,75 \mathrm{bc}(29,92)$ \\
T4 & 60 & $53,06 \mathrm{ab}(50,13)$ & $63,46 \mathrm{ab}(48,06)$ \\
T5 & 40 & 80 & $60,73 \mathrm{ab}(49,80)$ & $83,78 \mathrm{a}(56,73)$ \\
T6 & 20 & 100 & $140,92 \mathrm{a}(73,00)$ & $144,19 \mathrm{a}(71,00)$ \\
\hline
\end{tabular}

Em que: médias seguidas pela mesma letra numa mesma coluna não diferem significativamente entre si pelo teste de Kruskal-Wallis. Valores entre parênteses referem-se a classificação das médias conforme Teste de Kruskal-Wallis.

Os valores de Absorção de Água acompanham o comportamento do Inchamento em Espessura. Tanto após 2 quanto 24 h os painéis aglomerados fabricados com lignosulfonato puro apresentaram os maiores valores de Absorção de Água.

Tanto após 2 h quanto após 24 h de imersão em água, não houve diferença significativa entre os tratamentos T1, T2 e T3, ou seja a substituição de até 40\% de fenol-formaldeído por lignosulfonato não alterou negativamente os valores de Absorção em Água.

Considerando os resultados obtidos por Çetin e Özmen (2003), que a 20\% de substituição de fenol-formaldeído obtiveram uma Absorção de Água de 65,8\% para 2 h e 73,2\% para 24 h, e a 30\% obtiveram 69,9\% para 2 h e 72,9\% para 24 h; observa-se que os valores em Absorção de Água são consideravelmente superiores ao encontrados neste estudo.

O fato de ter sido feita a utilização de solução hidróxido de sódio pode explicar elevados valores de Inchamento em Espessura e Absorção de Água para os tratamentos T4, T5 e T6, já que esta é uma solução altamente higroscópica, contribuindo para Absorção de Água nos painéis.

\section{Avaliação das propriedades mecânicas dos painéis}

Os resultados de Resistência à Tração Perpendicular (Ligação Interna) estão apresentados na Tabela 7.

Na propriedade de Ligação Interna dos painéis aglomerados verificou-se diferença estatística entre os tratamentos. Painéis com fenol-formaldeído puro (T1) e em substituição de $20 \%$ por lignosulfonato (T2) apresentaram os maiores valores médios, sem diferença significativa entre os dois, ou seja, ao nível de $20 \%$ de substituição a resistência se mantém a mesma do fenol-formaldeído puro. À medida que o adesivo fenol-formaldeído foi substituído por lignosulfonato em maiores proporções, houve uma diminuição dos valores de Ligação Interna, sendo a diferença significativa. 
Silva et al. - Utilização de lignosulfonato e adesivo Fenol-Formaldeído na produção de painéis aglomerados

Tabela 7. Valores médios de Ligação Interna dos painéis fabricados com substituição de lignosulfonato pelo adesivo fenol-formaldeído.

Table 7. Average values of internal bond panels manufactured with replacement lignosulphonate by adhesive phenol-formaldehyde.

\begin{tabular}{|c|c|c|c|}
\hline \multirow{2}{*}{ Tratamento } & \multicolumn{2}{|c|}{ Composição do Adesivo (\%) } & \multirow{2}{*}{ Ligação Interna $\left(\mathrm{N} / \mathrm{mm}^{2}\right)$} \\
\hline & FF & LS & \\
\hline T1 & 100 & 0 & 1,11 a $(9,96)$ \\
\hline T2 & 80 & 20 & $1,04 \mathrm{ab}(15,09)$ \\
\hline T3 & 60 & 40 & 0,96 b $(10,60)$ \\
\hline T4 & 40 & 60 & 0,82 с $(5,70)$ \\
\hline T5 & 20 & 80 & $0,57 \mathrm{~d}(16,77)$ \\
\hline T6 & 0 & 100 & 0,18 e $(23,81)$ \\
\hline
\end{tabular}

Em que: médias seguidas pela mesma letra não diferem significativamente entre si pelo teste Tukey no nível de $5 \%$ de significância. Valores entre parênteses referem-se ao Coeficiente de Variação.

Pode-se observar que os resultados obtidos foram satisfatórios ao atendimento da NBR14810 para todas as composições adesivas estudadas, exceto para o tratamento T6, que não alcançou o requisito mínimo exigido, demonstrando que o lignosulfonato de cálcio e magnésio possui alta resistência adesiva quando associado com fenol-formaldeído.

Os resultados podem ser considerados satisfatórios quando comparados aos valores obtidos por Trianoski et al. (2011) ao avaliarem a possibilidade de espécies de alto crescimento para a produção de painéis colados com o adesivo ureia-formaldeído. Os autores obtiveram valor de Ligação Interna de $1,20 \mathrm{~N} / \mathrm{mm}^{2}$ utilizando somente Pinus taeda, e 0,69 N/mm² ao utilizar Pinus taeda em conjunto com Grevillea robusta.

Rosa e Hillig (2014) obtiveram valor de Ligação Interna de $0,2 \mathrm{~N} / \mathrm{mm}^{2}$ para painéis produzidos com $100 \%$ de lignosulfonato de amônio, valor bem próximo ao encontrado neste estudo. Em painéis compostos de 50\% de lignosulfonato e 50\% de ureia-formaldeído os autores encontraram 0,3 $\mathrm{N} / \mathrm{mm}^{2}$, valor bem inferior se comparado com valores obtidos neste trabalho na substituição por $60 \%$ de lignosulfonato (T4), e até mesmo para o tratamento com $80 \%$ de substituição por lignosulfonato (T5).

Em substituição de fenol-formaldeído por lignina organosolv modificada, Çetin e Özmen (2003) encontraram valores médios de Ligação Interna iguais aos encontrados neste trabalho com $20 \%$ de substituição. Já para 30\%, os autores encontraram o mesmo valor médio que este estudo encontrou para $40 \%$ de substituição de lignosulfonato por fenol-formaldeído.

Os Módulos de Elasticidade (MOE) e de Ruptura (MOR) estão representados na Tabela 8.

Tabela 8. Valores médios de MOE e MOR dos painéis fabricados com substituição de lignosulfonato pelo adesivo fenol-formaldeído.

Table 8. Mean values of MOR and MOE of the boards manufactured with replacement lignosulphonate by phenol-formaldehyde adhesive.

\begin{tabular}{|c|c|c|c|c|}
\hline \multirow{2}{*}{ Tratamento } & \multicolumn{2}{|c|}{ Composição do Adesivo (\%) } & \multicolumn{2}{|c|}{ Flexão Estática $\left(\mathrm{N} / \mathrm{mm}^{2}\right)$} \\
\hline & FF & LS & MOR & MOE \\
\hline T1 & 100 & 0 & 26,02 a $(17,74)$ & 3032 a $(24,85)$ \\
\hline T2 & 80 & 20 & $21,68 \mathrm{ab}(20,83)$ & 2714 a $(15,70)$ \\
\hline T3 & 60 & 40 & 24,99 a $(16,70)$ & 3304 a $(19,87)$ \\
\hline T4 & 40 & 60 & $22,89 \mathrm{ab}(9,64)$ & 3100 a $(7,21)$ \\
\hline T5 & 20 & 80 & 18,07 b $(24,26)$ & 2728 a $(18,85)$ \\
\hline T6 & 0 & 100 & 5,81 c $(36,17)$ & 1469 b $(19,36)$ \\
\hline
\end{tabular}

Em que: médias seguidas pela mesma letra numa mesma coluna não diferem significativamente entre si pelo teste Tukey no nível de $5 \%$ de significância. Valores entre parênteses referem-se ao Coeficiente de Variação.

Os maiores valores de MOR foram encontrados no T1, mas não houve diferença significativa entre os tratamentos T1, T2, T3 e T4. Assim, a substituição de até $60 \%$ do adesivo fenol-formaldeído por lignosulfonato não acarretou alteração negativa nos valores de MOR. A partir de $80 \%$ de substituição de fenol-formaldeído por lignosulfonato (T5) e nos painéis produzidos com lignosulfonato puro (T6) os valores de MOR decrescem, em função também dos baixos valores de sólidos contidos nos adesivos. 
Em 20\% de substituição de fenol-formaldeído por lignina organosolv modificada, Çetin e Özmen (2003) encontraram valor médio de MOR de $16 \mathrm{~N} / \mathrm{mm}^{2}$, para 30\% este valor diminuiu para $12,7 \mathrm{~N} / \mathrm{mm}^{2}$. Assim, os valores encontrados neste trabalho foram substancialmente superiores em comparação com os autores mencionados.

Para MOE, não houve diferença significativa nos painéis fabricados com adesivo fenol-formaldeído e suas diferentes misturas, exceto para o T6.

Para as análises de MOE, quando comparados à substituição de $20 \%$ por lignosulfonato, Cerquera (2010) encontrou valores relativamente superiores para painéis fabricados com substituição de $20 \%$ e $25 \%$, sendo os valores, respectivamente, $3954 \mathrm{~N} / \mathrm{mm}^{2}$ e $4276 \mathrm{~N} / \mathrm{mm}^{2}$. No entanto, a $30 \%$ de substituição, o valor obtido pelo autor sofreu um decréscimo substancial, passando para $1466,65 \mathrm{~N} / \mathrm{mm}^{2}$.

Para os painéis fabricados com 100\% de lignosulfonato, os valores médios de MOE encontrados foram ligeiramente inferiores aos obtidos por Rosa e Hillig (2014), ao encontrarem o valor de 1669 $\mathrm{N} / \mathrm{mm}^{2}$ utilizando lignosulfonato de amônio. Em substituição de 50\% de ureia-formaldeído por lignosulfonato, os autores encontraram valores médios de MOE de 2152,8 N/mm², valores inferiores aos obtidos neste trabalho com $80 \%$ de substituição de fenol-formaldeído por lignosulfonato, que foram de $2728 \mathrm{~N} / \mathrm{mm}^{2}$.

Çetin e Özmen (2003) obtiveram para 20\% e 30\% de substituição por lignina organosolv modificada, os valores $2267 \mathrm{~N} / \mathrm{mm}^{2}$ e $2224 \mathrm{~N} / \mathrm{mm}^{2}$, o que demonstra claramente desempenho inferior a este estudo.

\section{CONCLUSÕES}

As densidades básica e aparente da madeira de Pinus caribaea apresentaram valores considerados dentro da faixa ideal para produção de painéis aglomerados, não influenciando significativamente no desempenho dos tratamentos.

A substituição do adesivo fenol-formaldeído por lignosulfonato em até $80 \%$ foi satisfatória no atendimento a norma específica para propriedades mecânicas.

Não é possível produzir painéis com propriedades mínimas de resistência com lignosulfonato puro.

A higroscopicidade do lignosulfonato diluído em solução de hidróxido de sódio não permite que, ao analisar as propriedades de Inchamento em Espessura e Absorção de Água, acima de 20\% de substituição de fenol-formaldeído a NBR 14810 (ABNT, 2013) seja atendida.

O tempo de prensagem é o fator determinante para a utilização do lignosulfonato como adesivo na fabricação de painéis aglomerados.

O aumento da temperatura só poderá influenciar favoravelmente a utilização do lignosulfonato se o tempo mínimo de 10 minutos for respeitado para a completa cura do mesmo.

\section{REFERÊNCIAS BIBLIOGRÁFICAS}

ABNT - ASSOCIAÇÃO BRASILEIRA DE NORMAS TÉCNICAS. NBR 14810-2: Painéis de partículas de média densidade. Parte 2: Requisitos e métodos de ensaio. São Paulo, 69 p, 2013.

ALEMDAR, A.; SAIN, M. Biocomposites from wheat straw nanofibers: Morphology, thermal and mechanical properties. Composites Science and Technology, v. 68, n. 2, p. 557-565, 2008.

CABRAL, C. P.; VITAL, B. R.; DELLA LUCIA, R.M.; PIMENTA, A. S. Propriedades de chapas de aglomerado confeccionadas com misturas de partículas de Eucalyptus spp. e Pinus elliottii. Revista Árvore, Viçosa, v. 31, n. 5, p. 897-905, 2007.

CERQUERA, N. S. Estudio de la sustitución parcial del fenol por lignina en la obtención de las resinas fenol- formaldehído utilizadas para la fabricación de tableros aglomerados OSB a partir de especies forestales colombianas. 2010. 66 p. Trabajo de grado (Química Industrial) - Universid Tecnológica de Pereira, Colômbia, 2010. 
Silva et al. - Utilização de lignosulfonato e adesivo Fenol-Formaldeído na produção de painéis aglomerados

ÇETIN, N. S.; ÖZMEN E. Studies on Lignin-Based Adhesives for Particleboard Panels. Turkish Journal of Agriculture and Forestry, Ankara, v. 27, p. 183-189. 2003.

CHOW, S. Adhesive developments in Forest products. Wood Science and Technology, New York. v. 17, n. 1, p. 1-11, 1983.

DONATI, R. D. Produção de painéis reconstituídos no Brasil - passado, presente e futuro. 2010. Disponível em: < http://www.abipa.org.br/entrevistaRosane.php >. Acesso em: 06 jun. 2014.

FORSS, K. G., FUHRMANN, A. Finnish plywood, particleboard, and fireboard made with a lignin-base adhesive. Forest Products Journal, Madison, v. 29, n. 7, p. 39-43,1979.

HILLIG, E. Qualidade de chapas aglomeradas estruturais, fabricadas com madeiras de pinus, eucalipto e acácia negra, puras ou misturadas, coladas com tanino-formaldeído. 2000, 112 p. Dissertação (Mestrado em Engenharia Florestal) - Universidade Federal de Santa Maria, Santa Maria, 2000.

IWAKIRI, S.; CAPRARA, A. C.; SAKS, D. C. O.; PRADO, F.; FRANZONI, J. A.; KRAMBECK, L. B. P.; RIGATTO, P. A. Produção de painéis de madeira aglomerada de alta densificação com diferentes tipos de resina. Scientia Forestalis, Piracicaba, n. 68, p. 39-43, 2005.

IWAKIRI, S.; SILVA, J. R. M.; MATOSKI, S. L. S.; LEONHAD, G.; CARON, J. Produção de chapas de madeira aglomerada de cinco espécies de pinus tropicais. Floresta e Ambiente, Seropédica, v. 8, n.1, p.137-142, 2001.

MALONEY, T.M. Modern particleboard and dry-process fiberboard manufacturing. 2. ed. San Francisco: Miller Freeman, 1993. 689 p.

MATTOS, R. L.; GONÇALVES, R. M.; CHAGAS, F. B. Painéis de madeira no Brasil: panorama e perspectivas. BNDS Setorial, Rio de Janeiro, v. 27, p. 121 - 156, 2008.

MELO, R. R.; SANTINI, E. J.; HASELEIN, C. R. STANGERLIN, D. M.; MULLER, M. T.; DEL MENEZZI, C. H. S. Avaliação das propriedades físico-mecânicas de painéis aglomerados de Eucalyptus grandis colados com uréiaformaldeído e tanino -formaldeído. Revista Floresta, Curitiba, v. 40, n. 3, p. 497-506, 2010.

MOSLEMI, A. A. Particleboard: materials. London: Southern University Press, 1974. v.1, p. 244.

PIZZI, A.; MITTAL, K. L. Handbook of Adhesive Technology. New York: Marcel Dekker, 1994. 1036 p.

REMADE. Mercado estimula produtos de madeira com valor agregado. Revista da Madeira, Curitiba, v. 14, n. $84,2004$.

RODRÍGUEZ, J. M. Estudio del curado de resinas lingo-novolacas. Propriedades termo-mecánicas de los produtos curados. 2005. 327 p. Tese (Doutorado em Ciencias Químicas) - Facultad de Ciencias Químicas Departamento de Ingeniería Química, Universidad Complutense de Madrid, Madrid, 2005.

ROFFAEL, E. Die Formaldehydabgabe von Spanplatten und anderen Holzwerkstoffen. Stuttgart: DRW-Verlag, 1982. $154 \mathrm{p}$.

ROSA, T. S.; HILLIG E. Utilização de lignosulfonato de amônio na produção de painéis aglomerados. Ciência da Madeira, Pelotas, v. 5, n. 2, p. 93-102, 2014.

SILVA, G. C. Qualidade de painéis aglomerados produzidos com adesivos à base de lignosulfonato e ureiaformaldeído. 2015. 95 p. Tese (Doutorado em Ciência e Tecnologia da Madeira) - Universidade Federal Rural do Rio de Janeiro, Rio de Janeiro, 2015. 
TRIANOSKI, R.; IWAKIRI, S.; MATOS J. L. M.; PRATA, J. G. Avaliação de espécies alternativas de rápido crescimento para produção de painéis de madeira aglomerada de três camadas. Scientia Forestalis, Piracicaba, v. 39, n. 89, p. 097-104, 2011.

VITAL, B. R. Métodos de determinação da massa específica da madeira. Viçosa: SIF, 1984, 21p. (Boletim Técnico) 
